\title{
Study of Cold Stress on the Germination and Seedling Stage and Determination of Recovery in Rice Varieties
}

\author{
Ali Soleymani $^{1}$ \& Mohamad Hesam Shahrajabian ${ }^{1}$ \\ ${ }^{1}$ Department of Agronomy and Plant Breeding, Khorasgan Branch, Islamic Azad University, Esfahan, Iran \\ Correspondence: Department of Agronomy and Plant Breeding, Khorasgan Branch, Islamic Azad University, \\ Esfahan, PO Box 81595-158, Iran. E-mail: a_Soleymani@khuisf.ac.ir
}

Received: June 14, 2012

Accepted: July 19, 2012 Online Published: August 22, 2012

doi:10.5539/ijb.v4n4p23

URL: http://dx.doi.org/10.5539/ijb.v4n4p23

\begin{abstract}
Rice is the second most important crop in the world after wheat, with more than 90 per cent grown in Asia. In order to evaluate the effects of cold stress in the period of germination and seedling and recovery percent of rice cultivars, two independent tests were performed in 2006-2007 in Rice Research Institution at Amol city. An experiment was conducted by split split plots within a randomized block design (CRD) with 54 caring composition, 3 replications and 6 cultivars were used. In this study, main plots were temperature, sub plots were length of stress and sub sub plots were cultivars. The levels of temperature including 10 oc (intense stress), $14^{\circ} \mathrm{C}$ (critical temperature) and $32^{\circ} \mathrm{C}$ (improving temperature, control) and levels of stress time $12,24,48$ hours and cultivars of local Tarom, Domsiya, Hybrid, Nemat, Gerde, Unda were selected two by two based on quality of product and then performed in unit area and finally resistance against cold respectively. On the period of germination with decreasing temperature from $32^{\circ} \mathrm{C}$ to $14^{\circ} \mathrm{C}$ and from $14^{\circ} \mathrm{C}$ to $10^{\circ} \mathrm{C}$, total decrease studied indices with exception of percentage of damage to seed are meaningful and similar reaction in recovery section of germination is obvious. In the period of seedling, we can see a meaningful decrease in significant level $1 \%$ about of decreasing temperature of total stress, percentage of damage to seedling, vigour of seedling. By increasing temperature in the period of stress of germination, there is a meaningful increase level $1 \%$ of total studied features with the exception of damage of seed. In the period of recovery of germination, there is a meaningful increase in significant level $1 \%$ of total studied features with the exception of damage to seed. In the period of stress of germination, there is a meaningful decrease at total features with the exception of sensitive to stress, percentage of damage to seedling and vigour of seedling and aceous recovery are similar in the period of seedling. By decreasing time of stress in the period of germination, there is a meaningful decrease by percent of damage total features with exception of rate of germination, percentage of germination. In the period of germination recovery, there is a meaningful increase in length if radicle, length of stem, percentage of germination and rate of germination and other features have meaningful decrease. In the period of seedling recovery, by decreasing time of stress, length of shoot, length of radicle, weight of wet, dry root and other features have meaningful increase. The effects of cultivar in total tests and stages, was significant level $1 \%$. The results show that, feature of germination and length of stem and percentage of damage to seed in different cultivars are various. In the period of recovery, percentage of germination, rate of germination and percentage of damage to seeds, had correct criteria for selecting cultivars sensitive to cold stress. Low temperature can delay pitch and growth of transplant for more time. We can use height of shoot in rice as an index for determining of cultivars resistant to cold. According to these results, we decide precisely for re cultivate rice store or selecting to resistant cultivar by introducing Nemat cultivar in the period of germination and hybrid cultivar in the period of seedling aceous as resistant cultivar during occurring stress of cold.
\end{abstract}

Keywords: rice, cold stress, germination, seedling, recovery percent

\section{Introduction}

The seed emergence, growth, development and spatial distribution of plants are severely restricted by a variety of environmental stresses (Ahmad et al., 2009). The cultivation of many important crops in Iran such as rice as main crop has been originated from generation to generation. Rice is one of important crop and it is a major contributor to human food supply (Singh et al., 2002; Albuquerque \& Carvalho, 2003; Feng et al., 2004; Vivek et al., 2004; Yoon et al., 2004; Li et al., 2009; Dou et al., 2011). It is the second most important cereal crop after 
wheat in the world (Mohanty \& Painuli, 2004; Mahadi et al., 2006). Raja Babu et al. (2005) reported that rice accounting for about 29 per cent of the total calorie in terms of food intake. Plants that emerge early contribute more to crop yield than those that emerge later (Gan et al., 1992). Dormancy is a genetically complex trait controlled by polygenes with effects modified by the genetic background and environmental factors (Rathi et al., 2011). Seeds exposed to unfavorable environmental conditions may have compromise the seedling establishment (Abdulrahmani et al., 2007). In the past two decades, intensive research has been conducted to improve seed germination and seedling emergence under unfavorable condition (Abdulrahmani et al., 2007). Desirable crop yields are achieved by proving seeds with an environment that encourages early germination and emergence (Mohanty \& Painuli, 2004). There is a little information on emergence of rice seedling under cold stress treatments. So, study of cold stress on the germination and seedling stage and determination of recovery in rice varieties in this study was attempted.

\section{Materials and Methods}

The study was conducted during 2006-2007, at Rice Research Institution in Amol city in the north of Iran. An experiment was conducted by split split plots within a randomized block design (CRD) with 54 caring composition, 3 replications and 6 cultivars were used. In this study, main plots were temperature, sub plots were length of stress and sub sub plots were cultivars. The levels of temperature including $10 \mathrm{oc}$ (intense stress), $14^{\circ} \mathrm{C}$ (critical temperature) and $32^{\circ} \mathrm{C}$ (improving temperature, control) and levels of stress time 12, 24, 48 hours and cultivars of local Tarom, Domsiya, Hybrid, Nemat, Gerde, Unda were selected two by two based on quality of product and then performed in unit area and finally resistance against cold respectively. All lots were of commercial seed conditioning size, as they were when initially obtained from the supplier. The first experiment was done at germination stage, in this stage, germination rate, germination percentage, radicle and coleoptile length, fresh radicle and coleoptile weight, dry radicle and coleoptile weight and percentage of seed damage was evaluated. At first 40 uniform seeds 7 were sterilized, and placed in sterilized Petri dishes with two Whatman No. 1 filter papers (These Whatman papers were sterilized in $20^{\circ} \mathrm{C}$ for 20 minutes). Then in each Petri dish, $3 \mathrm{~mm}$ distilled water added, after that one $\mathrm{mm}$ of distilled water was added to Petri dish every day. These Petri dishes were placed into the incubator of $32^{\circ} \mathrm{C}$ for 24 hours. After that time, 25 seeds of each cultivar in Petri dish with uniform germination status selected and other seeds were omitted. Then, these Petri dishes placed into the cold incubator. Average germinated rate was calculated according the following formula (Saeidi et al., 2008). GR= $\mathrm{N}_{1} / \mathrm{D}_{1}+\mathrm{N}_{2} / \mathrm{D}_{2}+\ldots .+\mathrm{N}_{\mathrm{n}} / \mathrm{D}_{\mathrm{n}}$, where $\mathrm{N}_{1}=$ number of germinated seeds in the first day, $\mathrm{N}_{\mathrm{n}}=$ number of germinated seeds in the final day, $\mathrm{D}_{1}=$ the first day of counting, $\mathrm{D}_{\mathrm{n}}=$ final day of counting. For evaluation germination percentage, the normal seeds (seeds that have normal coleoptile and radicle longer than $2 \mathrm{~mm}$ length) counted and changed into percentage. All seedlings that had complete morphology without lesions or defects were selected and evaluated for radicle and coleoptile length. The roots and stem after measuring for fresh weight, were washed with distilled water and dried in oven at $75^{\circ} \mathrm{C}$ for 24 hours. Seed damage percentage was also determined. In the second experiment, for determining the effect of cold stress on seedlings of rice another experiment was done in green house with the use of phytotron. Soil experiment was clay- Loam, $\mathrm{PH}=6.79$ and $\mathrm{EC}=0.896 \mathrm{ds} / \mathrm{m}$, and seedlings were transplanted at a depth of about $2 \mathrm{~cm}$. The $\mathrm{N}, \mathrm{P}_{2} \mathrm{O}_{5}$ and $\mathrm{K}_{2} \mathrm{O}$ were added 0.5 , 0.5 and $1 \mathrm{~g} / \mathrm{kg}$ of soil, respectively. All plots were irrigated immediately after sowing and subsequent irrigations were carried out daily until 3 leaves stage. After 3 and 4 leaves stage, the samples were taken to phytotron to begin new treatments on them. Seedling vegetative vigor and standard evaluation system for rice on the basis of SES recipe was determined. Stress susceptibility index for rice was calculated according the following formulae. At first stress intensity (SI) was determined and then SSI was evaluated.

$$
\begin{array}{r}
S I=1-\frac{\overline{Y_{S}}}{Y_{P}} \\
S S I=\frac{1-\frac{Y_{S}}{Y_{P}}}{S I}
\end{array}
$$

$\bar{Y}_{P}=$ Average yield of all cultivars in normal environment.

$\bar{Y}_{S}=$ Average yield of all cultivars in stress environment.

$Y_{P}=$ Average yield of all cultivars in normal environment.

$Y_{S}=$ Average yield of all cultivars in stress environment.

In all two experiments, the recovery in rice varieties was evaluated. Analysis of variance (ANOVA) was used to 
determine the significant differences. The Duncan's Multiple Range Test performed the separation. All statistics was performed with MSTAT-C program (version 2.10).

\section{Results and Discussion}

\subsection{Period of Being Germination in Stress Condition}

Temperature had significant effect on coleoptile and redicle length, fresh coleoptile and radicle weight, dry coleoptile and radicle length, seed damage percentage and germination rate in a period of being germination in stress condition. All experimental parameters were significantly increased from 10 to $32{ }^{\circ} \mathrm{C}$. Treat with $32{ }^{\circ} \mathrm{C}$ in all experimental characteristics had significant differences with other treatments. All experimental parameters were significantly affected by time in a period of being germination in stress condition (Table 1). Coleoptile and radicle length, fresh coleoptile and radicle weight, dry coleoptile and radicle weight, germination percentage, seed damage percentage and germination rate were significantly increased from 12 to 48 hours. There were significant differences with three treatments in a period of being germination stress condition. All experimental characteristics also significantly affected by cultivar. The maximum coleoptile and radicle length, fresh coleoptile and radicle weight, dry coleoptile and radicle weight was obtained in Nemat cultivar in a period being germination in stress condition. The minimum and maximum germination percentage was $81.4 \%$ and $86.66 \%$, and it was obtained in Domsiya and Nemat, respectively. Domsiya had obtained the maximum seed damage percentage and the minimum one was related to Unda. Nemat and Unda, both had obtained the maximum germination rate. The differences between Unda and Nemat in a period of being germination in stress condition was not significant, but they have significant differences with other cultivars (Table 1).

Table 1. Mean comparison for coleoptile length $(\mathrm{mm})$, radicle length $(\mathrm{mm})$, fresh coleoptile weight $(\mathrm{mg})$, fresh radicle length $(\mathrm{mg})$, dry coleoptile weight $(\mathrm{mg})$, dry radicle weight $(\mathrm{mg})$, germination percentage $(\%)$, seed damage (\%), germination rate in a period of being germination in stress condition

\begin{tabular}{|c|c|c|c|c|c|c|c|c|c|}
\hline Treatment & $\begin{array}{c}\text { Coleoptile } \\
\text { length }\end{array}$ & $\begin{array}{l}\text { Radicle } \\
\text { length }\end{array}$ & $\begin{array}{c}\text { Fresh } \\
\text { coleoptile } \\
\text { weight }\end{array}$ & $\begin{array}{l}\text { Fresh } \\
\text { radicle } \\
\text { weight }\end{array}$ & $\begin{array}{c}\text { Dry } \\
\text { coleoptile } \\
\text { weight }\end{array}$ & $\begin{array}{c}\text { Dry } \\
\text { radicle } \\
\text { weight }\end{array}$ & $\begin{array}{c}\text { Germination } \\
\text { percentage }\end{array}$ & $\begin{array}{c}\text { Seed } \\
\text { damage } \\
\text { percentage }\end{array}$ & $\begin{array}{c}\text { Germination } \\
\text { rate }\end{array}$ \\
\hline \multicolumn{10}{|l|}{$\begin{array}{l}\text { Temperature } \\
\left({ }^{\circ} \mathrm{C}\right)\end{array}$} \\
\hline 10 & $1.324 \mathrm{c}$ & $2.888 \mathrm{c}$ & $0.721 \mathrm{c}$ & $1.087 \mathrm{c}$ & $0.163 \mathrm{c}$ & $0.168 \mathrm{c}$ & $81.08 \mathrm{c}$ & $86.04 \mathrm{a}$ & $108.6 \mathrm{c}$ \\
\hline 12 & $2.842 b$ & $7.784 \mathrm{~b}$ & $3.244 \mathrm{~b}$ & $3.279 \mathrm{~b}$ & $0.391 b$ & $0.422 b$ & $83.44 \mathrm{~b}$ & $83.68 b$ & $113.9 \mathrm{~b}$ \\
\hline 32 & $10.09 \mathrm{a}$ & $21 / 72 \mathrm{a}$ & $8.946 \mathrm{a}$ & $6.814 \mathrm{a}$ & $1.758 \mathrm{a}$ & $1.858 \mathrm{a}$ & $88.36 \mathrm{a}$ & $78.78 \mathrm{c}$ & $123.1 \mathrm{a}$ \\
\hline \multicolumn{10}{|l|}{$\begin{array}{l}\text { Time } \\
\text { (hours) }\end{array}$} \\
\hline 12 & $2.981 \mathrm{c}$ & $6.764 \mathrm{c}$ & $2.386 \mathrm{c}$ & $2.182 \mathrm{c}$ & $0.539 \mathrm{c}$ & $0.42 \mathrm{c}$ & $83.9 \mathrm{c}$ & $83.23 \mathrm{a}$ & $135.3 \mathrm{a}$ \\
\hline 24 & $4.338 \mathrm{~b}$ & $10.03 b$ & $3.512 b$ & $3.293 b$ & $0.652 b$ & $0.981 \mathrm{~b}$ & $84.29 b$ & $82.84 \mathrm{~b}$ & $113.5 \mathrm{~b}$ \\
\hline 48 & $6.934 \mathrm{a}$ & $15.69 \mathrm{a}$ & $7.013 \mathrm{a}$ & $5.656 a$ & $1.122 \mathrm{a}$ & $1.047 \mathrm{a}$ & $84.69 \mathrm{a}$ & $82.44 \mathrm{c}$ & $105.9 \mathrm{c}$ \\
\hline \multicolumn{10}{|l|}{ Cultivar } \\
\hline $\begin{array}{l}\text { Local } \\
\text { Tarom }\end{array}$ & $3.160 \mathrm{e}$ & $6.983 \mathrm{e}$ & $2.771 \mathrm{~d}$ & $1.986 \mathrm{e}$ & $0.474 \mathrm{e}$ & $0.437 \mathrm{e}$ & $81.9 \mathrm{e}$ & $85.25 b$ & $96.43 \mathrm{~d}$ \\
\hline Domsiya & $0.997 \mathrm{f}$ & $2.618 \mathrm{f}$ & $0.734 \mathrm{e}$ & $1.252 \mathrm{f}$ & $0.11 \mathrm{f}$ & $0.211 \mathrm{f}$ & $81.4 \mathrm{f}$ & $85.74 \mathrm{a}$ & $96.11 \mathrm{e}$ \\
\hline Hybrid & $4.975 \mathrm{c}$ & $13.48 \mathrm{c}$ & $3.132 \mathrm{~d}$ & $3.299 \mathrm{c}$ & $0.841 \mathrm{c}$ & $0.647 \mathrm{~d}$ & $84.85 \mathrm{c}$ & $82.27 \mathrm{~d}$ & $131.5 \mathrm{~b}$ \\
\hline Nemat & $7.695 \mathrm{a}$ & $14.32 \mathrm{~b}$ & $7.984 \mathrm{a}$ & $7.093 \mathrm{a}$ & $1.589 \mathrm{a}$ & $1.158 \mathrm{~b}$ & $86.66 \mathrm{~b}$ & $80.47 \mathrm{e}$ & $131.9 \mathrm{a}$ \\
\hline Gerde & $4.316 \mathrm{~d}$ & $11.3 \mathrm{~d}$ & $3.348 b$ & $2.516 \mathrm{~d}$ & $0.58 \mathrm{~d}$ & $1.339 \mathrm{a}$ & $84 d$ & $83.12 \mathrm{c}$ & $121.4 \mathrm{c}$ \\
\hline Unda & $7.363 b$ & $16.26 \mathrm{a}$ & $7.853 \mathrm{a}$ & $6.116 \mathrm{~b}$ & $1.03 \mathrm{~b}$ & $1.103 \mathrm{c}$ & $86.96 a$ & $80.17 \mathrm{f}$ & $131.9 \mathrm{a}$ \\
\hline
\end{tabular}

Common letters within each column do not differ significantly.

\subsection{Period of Being Germination in a Recovery Stage}

Temperature had significant influence on all experimental characteristics. All experimental characteristics were 
significantly increased by increasing temperature, but the highest seed damage percentage was obtained in 10 and $12{ }^{\circ} \mathrm{C}$, and there were no significant differences in seed damage percentage between three treatments (Table 2). Time also had significant influence on coleoptile and radicle length, fresh coleoptile and radicle weight, dry coleoptile and radicle weight, germination percentage, seed damage percentage and germination rate. The maximum of coleoptile and radicle length, fresh coleptile and radicle weight, dry coleoptile and radicle weight was related to $40{ }^{\circ} \mathrm{C}$. The maximum coleoptile and radicle length was obtained in Gerde cultivar. Hybrid and Unda had obtained the highest fresh coleoptile weight and fresh radicle weight, respectively. The maximum dry coleoptile and radicle weight was achieved in Hybrid cultivar. Local Tarom, Hybrid, Nemat, Gerde and Unda had no significant difference in germination percentage with eachother, but all of these cultivars had significant difference with Domsiya cultivar. The highest seed damage percentage was achieved in Domsiya cultivar, and this cultivar had significant difference with other cultivars. Unda and Domsiya had obtained the maximum and minimum germination rate, respectively (Table 2 ).

Table 2. Mean comparison for coleoptile length $(\mathrm{mm})$, radicle length $(\mathrm{mm})$, fresh coleoptile weight $(\mathrm{mg})$, fresh radicle length $(\mathrm{mg})$, dry coleoptile weight $(\mathrm{mg})$, dry radicle weight $(\mathrm{mg})$, germination percentage $(\%)$, seed damage (\%), period of being germination in a recovery stage

\begin{tabular}{|c|c|c|c|c|c|c|c|c|c|}
\hline Treatment & $\begin{array}{l}\text { Coleoptile } \\
\text { length }\end{array}$ & $\begin{array}{c}\text { Radicle } \\
\text { length }\end{array}$ & $\begin{array}{c}\text { Fresh } \\
\text { coleoptile } \\
\text { weight }\end{array}$ & $\begin{array}{l}\text { Fresh } \\
\text { radicle } \\
\text { weight }\end{array}$ & $\begin{array}{c}\text { Dry } \\
\text { coleoptile } \\
\text { weight }\end{array}$ & $\begin{array}{c}\text { Dry } \\
\text { radicle } \\
\text { weight }\end{array}$ & $\begin{array}{c}\text { Germination } \\
\text { percentage }\end{array}$ & $\begin{array}{c}\text { Seed } \\
\text { damage } \\
\text { percentage }\end{array}$ & $\begin{array}{l}\text { Germination } \\
\text { rate }\end{array}$ \\
\hline \multicolumn{10}{|l|}{$\begin{array}{c}\text { Temperature } \\
\left({ }^{\circ} \mathrm{C}\right)\end{array}$} \\
\hline 10 & $27.74 \mathrm{c}$ & $55.21 \mathrm{c}$ & $26.87 \mathrm{c}$ & $27.44 \mathrm{c}$ & $3.441 \mathrm{c}$ & $3.206 \mathrm{c}$ & $88.5 \mathrm{~b}$ & $108.9 a$ & $55.52 \mathrm{c}$ \\
\hline 12 & $31.76 \mathrm{~b}$ & $60.61 \mathrm{~b}$ & $31.57 \mathrm{~b}$ & $30.65 b$ & $4.199 \mathrm{~b}$ & $3.694 \mathrm{~b}$ & $88.61 \mathrm{a}$ & $108.9 \mathrm{a}$ & $56.23 \mathrm{~b}$ \\
\hline 32 & $45.59 \mathrm{a}$ & $73.86 \mathrm{a}$ & $45.54 \mathrm{a}$ & $46.98 \mathrm{a}$ & $8.715 \mathrm{a}$ & $6.731 \mathrm{a}$ & $88.61 \mathrm{a}$ & $108.7 \mathrm{~b}$ & $61.75 \mathrm{a}$ \\
\hline \multicolumn{10}{|l|}{$\begin{array}{l}\text { Time } \\
\text { (hours) }\end{array}$} \\
\hline 12 & $37.7 \mathrm{a}$ & $66.84 \mathrm{a}$ & $30.94 b$ & $33.02 \mathrm{~b}$ & $4.578 \mathrm{c}$ & $4.158 \mathrm{~b}$ & $88.59 \mathrm{a}$ & $108.8 \mathrm{~b}$ & $63.94 a$ \\
\hline 24 & $36.18 \mathrm{~b}$ & $64.19 \mathrm{~b}$ & $31.55 b$ & $32.73 b$ & $4.845 b$ & $4.199 \mathrm{~b}$ & $88.58 \mathrm{ab}$ & $108.8 \mathrm{a}$ & $57.41 \mathrm{~b}$ \\
\hline 48 & $31.2 \mathrm{c}$ & $58.65 \mathrm{c}$ & $41.48 \mathrm{a}$ & $39.31 \mathrm{a}$ & $6.933 \mathrm{a}$ & $5.275 \mathrm{a}$ & $88.55 b$ & $108.7 \mathrm{a}$ & $52.16 \mathrm{c}$ \\
\hline \multicolumn{10}{|l|}{ Cultivar } \\
\hline $\begin{array}{l}\text { Local } \\
\text { Tarom }\end{array}$ & $34.31 \mathrm{c}$ & $45.7 \mathrm{~d}$ & $29.1 \mathrm{~d}$ & $25.67 \mathrm{e}$ & $4.55 \mathrm{~d}$ & $4.28 \mathrm{c}$ & $88.61 \mathrm{a}$ & $108.4 \mathrm{~b}$ & $55.37 \mathrm{e}$ \\
\hline Domsiya & $27.06 \mathrm{~d}$ & $47.46 \mathrm{~d}$ & $28.25 \mathrm{~d}$ & $23.33 \mathrm{f}$ & $4.31 \mathrm{e}$ & $2.88 \mathrm{~d}$ & $88.4 \mathrm{~b}$ & $108.9 \mathrm{a}$ & $54.86 \mathrm{f}$ \\
\hline Hybrid & $33.79 \mathrm{c}$ & $78.37 \mathrm{a}$ & $40.27 \mathrm{a}$ & $40.69 \mathrm{~b}$ & $6.56 \mathrm{a}$ & $5.50 \mathrm{a}$ & $88.61 \mathrm{a}$ & $108.4 b$ & $58.61 \mathrm{c}$ \\
\hline Nemat & $37.22 b$ & $61.48 \mathrm{c}$ & $33.95 \mathrm{c}$ & $38.43 \mathrm{~d}$ & $5.63 \mathrm{c}$ & $5.42 \mathrm{a}$ & $88.61 \mathrm{a}$ & $108.4 b$ & $60.38 b$ \\
\hline Gerde & $39.04 \mathrm{a}$ & $78.51 \mathrm{a}$ & $36.64 \mathrm{~b}$ & $39.86 \mathrm{c}$ & $5.49 \mathrm{c}$ & $4.33 \mathrm{c}$ & $88.61 \mathrm{a}$ & $108.4 b$ & $56.91 \mathrm{~d}$ \\
\hline Unda & $38.75 \mathrm{a}$ & $67.84 \mathrm{~b}$ & $39.73 a$ & $42.15 \mathrm{a}$ & $6.14 \mathrm{~b}$ & $4.82 \mathrm{~b}$ & $88.61 \mathrm{a}$ & $108.4 \mathrm{~b}$ & $60.87 \mathrm{a}$ \\
\hline
\end{tabular}

Common letters within each column do not differ significantly.

\subsection{Period of Being Seedling in Stress Condition}

All experimental characteristics were affected by temperature. The highest aerial seedling length, root length, fresh aerial weight, fresh root weight, dry seedling weight, dry root weight was achieved in $32{ }^{\circ} \mathrm{C}$. The maximum stress susceptibility and seedling vigor was obtained in 12 and $10{ }^{\circ} \mathrm{C}$, respectively. Seedling damage percentage was significantly decreased from 10 to $32{ }^{\circ} \mathrm{C}$ (Table 3). Aerial seedling length, root length, fresh aerial weight, fresh root weight, dry seedling weight and dry root weight were significantly affected by time. In all these characteristics, the significant increase was found from 12 to 48 hours. The effect of time was not significant on stress susceptibility index, seedling vigor and seedling damage percentage, and there were no significant differences in these experimental characteristics between 12, 24 and 49 hours. The maximum aerial seedling and root length was achieved in Gerde cultivar. Gerde also had obtained the highest fresh aerial weight, and the 
difference between this cultivar and other cultivars were significant. The maximum fresh root weight was related to Hybrid. The maximum dry seedling weight and dry root weight was obtained in Gerde and Hybrid, respectively. Local Tarom had obtained the maximum stress susceptibility index, and the minimum one is related to Domsiya. Local Tarom also had obtained the maximum seedling vigor and seedling damage percentage, but the difference in seedling damage percentage between Local Tarom and Domsiya is not significant (Table 3).

Table 3. Mean comparison for aerial seedling length $(\mathrm{cm})$, root length $(\mathrm{cm})$, fresh aerial weight $(\mathrm{mg})$, fresh root weight $(\mathrm{mg})$, dry seedling weight $(\mathrm{mg})$, dry root weight $(\mathrm{mg})$, stress susceptibility index, seedling vigor and seedling damage percentage (\%) in a period of being seedling in stress condition

\begin{tabular}{|c|c|c|c|c|c|c|c|c|c|}
\hline Treatment & $\begin{array}{l}\text { aerial } \\
\text { seedling } \\
\text { length }\end{array}$ & $\begin{array}{l}\text { root } \\
\text { length }\end{array}$ & $\begin{array}{c}\text { fresh } \\
\text { aerial } \\
\text { weight }\end{array}$ & $\begin{array}{c}\text { fresh } \\
\text { root } \\
\text { weight }\end{array}$ & $\begin{array}{c}\text { dry } \\
\text { seedling } \\
\text { weight }\end{array}$ & $\begin{array}{l}\text { dry root } \\
\text { weight }\end{array}$ & $\begin{array}{c}\text { stress } \\
\text { susceptibility } \\
\text { index }\end{array}$ & $\begin{array}{l}\text { seedling } \\
\text { vigor }\end{array}$ & $\begin{array}{l}\text { seedling } \\
\text { damage } \\
\text { percentage }\end{array}$ \\
\hline \multicolumn{10}{|l|}{$\begin{array}{c}\text { Temperature } \\
\left({ }^{\circ} \mathrm{C}\right)\end{array}$} \\
\hline 10 & $15.07 \mathrm{c}$ & $5.58 \mathrm{c}$ & $133.7 \mathrm{c}$ & $57.17 \mathrm{c}$ & $26.13 c$ & $11.84 \mathrm{c}$ & $1.03 \mathrm{a}$ & $2.66 \mathrm{a}$ & $2.25 \mathrm{a}$ \\
\hline 12 & $16.28 \mathrm{~b}$ & $6.86 \mathrm{~b}$ & $171.2 \mathrm{~b}$ & $85.77 \mathrm{~b}$ & $34.02 \mathrm{~b}$ & $16.21 \mathrm{~b}$ & $1.17 \mathrm{a}$ & $2.59 \mathrm{a}$ & $2.20 \mathrm{a}$ \\
\hline 32 & $19.2 \mathrm{a}$ & $8.78 \mathrm{a}$ & $204.3 \mathrm{a}$ & $110.1 \mathrm{a}$ & $40.99 \mathrm{a}$ & $20.85 \mathrm{a}$ & $0 \mathrm{~b}$ & $1.19 b$ & $1 b$ \\
\hline \multicolumn{10}{|l|}{$\begin{array}{l}\text { Time } \\
\text { (hours) }\end{array}$} \\
\hline 12 & $16.05 \mathrm{c}$ & $6.53 \mathrm{c}$ & $159.1 \mathrm{c}$ & $74.86 \mathrm{c}$ & $31.28 \mathrm{c}$ & $14.31 \mathrm{c}$ & $0.67 \mathrm{a}$ & $2.15 \mathrm{a}$ & $1.80 \mathrm{a}$ \\
\hline 24 & $16.71 \mathrm{~b}$ & $6.97 \mathrm{~b}$ & $168.5 \mathrm{~b}$ & $82.75 b$ & $33.69 \mathrm{~b}$ & $16.05 b$ & $0.70 \mathrm{a}$ & $2.16 \mathrm{a}$ & $1.80 \mathrm{a}$ \\
\hline 48 & $17.19 \mathrm{a}$ & $7.72 \mathrm{a}$ & $181.6 \mathrm{a}$ & $96.46 \mathrm{a}$ & $36.18 \mathrm{a}$ & $18.53 \mathrm{a}$ & $0.76 a$ & $2.13 a$ & $1.85 \mathrm{a}$ \\
\hline \multicolumn{10}{|l|}{ Cultivar } \\
\hline $\begin{array}{l}\text { Local } \\
\text { Tarom }\end{array}$ & $17.35 \mathrm{c}$ & $7.03 \mathrm{c}$ & $146 \mathrm{c}$ & $82.51 \mathrm{c}$ & $30.9 \mathrm{c}$ & $14.97 \mathrm{~d}$ & $0.91 \mathrm{a}$ & $2.51 \mathrm{a}$ & $2.10 \mathrm{a}$ \\
\hline Domsiya & $12.68 \mathrm{f}$ & $6.35 \mathrm{~d}$ & $92.99 \mathrm{f}$ & $46.85 \mathrm{e}$ & $22.68 \mathrm{e}$ & $13.64 \mathrm{e}$ & $1.02 \mathrm{a}$ & $2.48 \mathrm{a}$ & $2.10 \mathrm{a}$ \\
\hline Hybrid & $20.03 b$ & $8.42 b$ & $263.4 \mathrm{a}$ & $125.7 \mathrm{a}$ & $45.86 \mathrm{e}$ & $19.58 \mathrm{a}$ & $0.43 b$ & $1.89 \mathrm{c}$ & $1.57 \mathrm{~cd}$ \\
\hline Nemat & $14.64 \mathrm{~d}$ & $5.72 \mathrm{f}$ & $138.2 \mathrm{~d}$ & $68.27 \mathrm{~d}$ & $27.03 \mathrm{~d}$ & $14.87 \mathrm{~d}$ & $0.46 \mathrm{~b}$ & $1.86 \mathrm{c}$ & $1.53 \mathrm{~d}$ \\
\hline Gerde & $22.54 \mathrm{a}$ & $8.86 a$ & $244.5 b$ & $100.5 b$ & $48.82 \mathrm{a}$ & $18.71 \mathrm{~b}$ & $0.56 \mathrm{~b}$ & $1.96 \mathrm{c}$ & $1.65 \mathrm{c}$ \\
\hline Unda & $13.86 \mathrm{e}$ & $6.05 \mathrm{e}$ & $133.5 \mathrm{e}$ & $84.32 \mathrm{c}$ & $84.32 \mathrm{c}$ & $16.01 \mathrm{c}$ & $0.89 a$ & $2.18 \mathrm{~b}$ & $1.95 \mathrm{~b}$ \\
\hline
\end{tabular}

Common letters within each column do not differ significantly.

\subsection{Period of Being Seedling in Recovery Stage}

In this period, the significant increase in aerial seedling length, root length, fresh aerial and root weight, dry seedling and root weight was achieved from 10 to $32{ }^{\circ} \mathrm{C}$. The minimum stress susceptibility index, seedling vigor and seedling damage percentage was related to $32{ }^{\circ} \mathrm{C}$. Expect of, stress susceptibility index and seedling damage percentage, all other characteristics influenced by time. The highest seedling vigor was obtained in 48 hours, and its difference with all other treatments, were significant. The effect of cultivar on stress susceptibility index was not significant, and no trend was found. The maximum seedling vigor and seedling damage percentage was related to Domsiya and Gerde, respectively (Table 2). The susceptibility index ranges and seedling vigor is presented in table number 5 and table number 6 , respectively. 
Table 4. Mean comparison for aerial seedling length $(\mathrm{cm})$, root length $(\mathrm{cm})$, fresh aerial weight $(\mathrm{mg})$, fresh root weight $(\mathrm{mg})$, dry seedling weight $(\mathrm{mg})$, dry root weight $(\mathrm{mg})$, stress susceptibility index, seedling vigor and seedling damage percentage $(\%)$ in a period of recovery

\begin{tabular}{|c|c|c|c|c|c|c|c|c|c|}
\hline Treatment & $\begin{array}{l}\text { aerial } \\
\text { seedling } \\
\text { length }\end{array}$ & $\begin{array}{l}\text { root } \\
\text { length }\end{array}$ & $\begin{array}{c}\text { fresh } \\
\text { aerial } \\
\text { weight }\end{array}$ & $\begin{array}{c}\text { fresh } \\
\text { root } \\
\text { weight }\end{array}$ & $\begin{array}{c}\text { dry } \\
\text { seedling } \\
\text { weight }\end{array}$ & $\begin{array}{l}\text { dry root } \\
\text { weight }\end{array}$ & $\begin{array}{c}\text { stress } \\
\text { susceptibility } \\
\text { index }\end{array}$ & $\begin{array}{l}\text { seedling } \\
\text { vigor }\end{array}$ & $\begin{array}{c}\text { seedling } \\
\text { damage } \\
\text { percentage }\end{array}$ \\
\hline \multicolumn{10}{|l|}{$\begin{array}{c}\text { Temperature } \\
\left({ }^{\circ} \mathrm{C}\right)\end{array}$} \\
\hline 10 & $17.64 \mathrm{c}$ & $6.60 \mathrm{c}$ & $185.6 \mathrm{c}$ & $94.72 \mathrm{c}$ & $40.68 c$ & $17.17 \mathrm{c}$ & $0.98 \mathrm{a}$ & $2.65 a$ & $2.24 \mathrm{a}$ \\
\hline 12 & $20.74 b$ & $8.28 b$ & $222.1 \mathrm{~b}$ & $131.3 b$ & $47.7 b$ & $23.27 \mathrm{~b}$ & $0.99 a$ & $2.62 \mathrm{a}$ & $2.24 \mathrm{a}$ \\
\hline 32 & $25.72 \mathrm{a}$ & $12.49 \mathrm{a}$ & $335.1 \mathrm{a}$ & $229.4 \mathrm{a}$ & $68.78 \mathrm{a}$ & $41.11 \mathrm{a}$ & $0 \mathrm{~b}$ & $1.16 \mathrm{~b}$ & $1 \mathrm{~b}$ \\
\hline \multicolumn{10}{|l|}{ Time (hour) } \\
\hline 12 & $21.5 \mathrm{ab}$ & $8.78 b$ & $252.6 \mathrm{a}$ & $153 b$ & $53.33 \mathrm{a}$ & $27.52 \mathrm{a}$ & $0.66 \mathrm{a}$ & $2.17 \mathrm{a}$ & $1.81 \mathrm{a}$ \\
\hline 24 & $21.07 \mathrm{~b}$ & $8.97 b$ & $245.2 b$ & $147.1 \mathrm{~b}$ & $52.47 \mathrm{a}$ & $26.18 b$ & $0.66 \mathrm{a}$ & $2.19 \mathrm{a}$ & $1.85 \mathrm{a}$ \\
\hline 48 & $21.54 \mathrm{a}$ & $9.61 \mathrm{a}$ & $245.1 \mathrm{~b}$ & $155.4 \mathrm{a}$ & $51.36 \mathrm{~b}$ & $27.85 \mathrm{a}$ & $0.65 a$ & $2.08 \mathrm{~b}$ & $1.81 \mathrm{a}$ \\
\hline Cultivar & & & & & & & & & \\
\hline $\begin{array}{l}\text { Local } \\
\text { Tarom }\end{array}$ & $23.65 b$ & $8.67 \mathrm{~cd}$ & $249.6 c$ & $107.8 \mathrm{e}$ & $60.57 \mathrm{a}$ & $21.56 \mathrm{e}$ & $0.65 \mathrm{a}$ & $2.16 \mathrm{~b}$ & $1.82 \mathrm{~b}$ \\
\hline Domsiya & $18.59 \mathrm{c}$ & $8.09 \mathrm{e}$ & $144.1 \mathrm{f}$ & $66.67 f$ & $32.39 \mathrm{e}$ & $13.97 f$ & $0.55 \mathrm{a}$ & $2.26 \mathrm{a}$ & $1.69 \mathrm{c}$ \\
\hline Hybrid & $23.19 b$ & $9.90 \mathrm{~b}$ & $326.8 \mathrm{a}$ & $194.6 b$ & $58.93 b$ & $33.25 \mathrm{~b}$ & $0.68 \mathrm{a}$ & $2.15 b$ & $1.88 \mathrm{ab}$ \\
\hline Nemat & $18.55 \mathrm{c}$ & $8.34 \mathrm{de}$ & $221.1 \mathrm{e}$ & $160.8 d$ & $56.08 \mathrm{c}$ & $26.78 \mathrm{~d}$ & $0.69 a$ & $2.10 \mathrm{~b}$ & $1.85 \mathrm{ab}$ \\
\hline Gerde & $25.22 \mathrm{a}$ & $10.81 \mathrm{a}$ & $308.2 b$ & $209.1 \mathrm{a}$ & $59.63 \mathrm{ab}$ & $31.05 \mathrm{c}$ & $0.70 \mathrm{a}$ & $2.16 \mathrm{~b}$ & $1.9 \mathrm{a}$ \\
\hline Unda & $19.01 \mathrm{c}$ & $8.90 \mathrm{c}$ & $235.9 \mathrm{~d}$ & $172 \mathrm{c}$ & $46.71 \mathrm{~d}$ & $36.49 \mathrm{a}$ & $0.68 \mathrm{a}$ & $2.10 \mathrm{~b}$ & $1.83 \mathrm{ab}$ \\
\hline
\end{tabular}

Common letters within each column do not differ significantly.

Table 5. Susceptibility index ranges for cultivars

\begin{tabular}{ccc}
\hline Cultivar & Susceptibility index & Type of resistance \\
\hline Local Tarom & 0.6545 & Resistant \\
Domsiya & 0.5514 & Resistant \\
Hybrid & 0.6829 & Resistant \\
Nemat & 0.6975 & Resistant \\
Gerde & 0.7022 & Resistant \\
Unda & 0.683 & Resistant \\
\hline
\end{tabular}

Table 6. Seedling vigor on the basis of SES in period of recovery

\begin{tabular}{ccc}
\hline Cultivar & Seedling status & Number \\
\hline Local Tarom & Very weak & 9 \\
Domsiya & Weak & 7 \\
Hybrid & Weak & 7 \\
Nemat & Weak & 7 \\
Gerde & Weak & 7 \\
Unda & weak & 7 \\
\hline
\end{tabular}




\section{Conclusions}

The demand for rice in general in growing with both the rich and the urban poor relying on it as a major source of calories (Kolawole et al., 2010; 2011). On the period of germination with decreasing temperature from $32^{\circ} \mathrm{C}$ to $14^{\circ} \mathrm{C}$ and from $14^{\circ} \mathrm{C}$ to $10^{\circ} \mathrm{C}$, total decrease studied indices with exception of percentage of damage to seed are meaningful and similar reaction in recovery section of germination is obvious. In the period of seedling, we can see a meaningful decrease in significant level $1 \%$ about of decreasing temperature of total stress, percentage of damage to seedling, vigor of seedling. By increasing temperature in the period of stress of germination, there is a meaningful increase level $1 \%$ of total studied features with the exception of damage of seed. In the period of recovery of germination, there is a meaningful increase in significant level $1 \%$ of total studied features with the exception of damage to seed. In the period of stress of germination, there is a meaningful decrease at total features with the exception of sensitive to stress, percentage of damage to seedling and vigor of seedling and aceous recovery are similar in the period of seedling. By decreasing time of stress in the period of germination, there is a meaningful decrease by percent of damage total features with exception of rate of germination, percentage of germination. In the period of germination recovery, there is a meaningful increase in length if radicle, length of stem, percentage of germination and rate of germination and other features have meaningful decrease. In the period of stress of seedling, there is a meaningful decrease at total features with exception of sensitive to stress, percentage of damage to seedling and vigor of seedling. In the period of seedling recovery, by decreasing time of stress, length of shoot, length of radicle, weight of wet, dry root and other features have meaningful increase. The effects of cultivar in total tests and stages, was significant level $1 \%$. The results show that, feature of germination and length of stem and percentage of damage to seed in different cultivars are various. In the period of recovery, percentage of germination, rate of germination and percentage of damage to seed, had correct criteria for selecting cultivars sensitive to cold stress. Low temperature can delay pitch and growth of transplant for more time. We can use height of shoot in rice as an index for determining of cultivars resistant to cold. According to these results, we decide precisely for re cultivate rice store or selecting to resistant cultivar by introducing Nemat cultivar in the period of germination and hybrid cultivar in the period of seedling aceous as resistant cultivar during occurring stress of cold. To meet the increasing demand, the importance of study of cold stress on germination and seedling growth of rice is undeniable.

\section{References}

Abdulrahmani, B., Ghassemi-Golezani, K., Valizadeh, M., \& Feizi Asl, V. (2007). Seed priming and seedling establishment of barley (Hordeum vulgare L.). Journal of Food, Agriculture and Environment, 5(3\&4), 179-184.

Ahmad, S., Ahmad, R., Ashraf, M. Y., Ashraf, M., \& Waraich, E. A. (2009). Sunflower (Helianthus annuus L.) response to drought stress at germination and seedling growth stages. Pak. J. Bot., 41(2), 647-654.

Albuquerque, F. M. C., \& de Carvalho, N. M. de. (2003). Effect of type of environmental stress on the emergence of sunflower (Helianthus annuus L.), soybean (Glycine max (L.) Merril) and maize (Zea mays L.) seeds with different levels of vigor. Seed Sci. Technol., 31, 465-467.

Dou, S., Wei, J., Cao, Y., \& Lan, J. (2011). Molecular characterization and preliminary functional analysis of cystatin OC-I in rice. Journal of Food, Agriculture and Environment, 9(2), 235-239.

Feng, Y. W., Yoshinaga, I., Shiratani, E., Hitomi, T., \& Hasebe, H. (2004). Characteristics and behavior of nutrients in a paddy field area equipped with a recycling irrigation system. Agricultural Water Management, 68, 47-60. http://dx.doi.org/10.1016/j.agwat.2004.02.012

Gan, Y., Stobe, E. H., \& Moes, J. (1992). Relative date of wheat seedling emergence and its impact on grain yield. Crop Science, 32, 1275-1281. http://dx.doi.org/10.2135/cropsci1992.0011183X003200050042x

ISTA Reports. (1985). International Rules for Seed Testing. Rules, Canad.

Kolawole, A., Idowu Oladele, O., \& Wakatsuki, T. (2010). Impact of Sawah system on rice based farming system in Nigeria. Journal of Food, Agriculture and Environment, 8(3\&4), 473-475.

Kolawole, A., Idowu Oladele, O., \& Wakatsuki. T. (2011). Profitability of different sawah rice production models within lowlands in Nigeria. Journal of Food, Agriculture and Environment, 9(1), 268-274.

Li, Y., Yang, L. Z., \& Wang. C. (2009). Evaluation of fertilizing schemes for direct-seeding rice fields in Taihu Lake Basin, China. Turkish Journal of Agriculture and Forestry, 34, 83-90.

Mahadi, M. A., Dadari, S. A., Mahmud, M., \& Babaji, B. A. (2006). Effect of pre-emergence herbicides on yield and yield components of rice. Journal of Food, Agriculture and Environment, 4(2), 164-167. 
Mohanty, M., \& Painuli, D. K. (2004). Modeling rice seedling emergence and growth under tillage and residue management in a rice-wheat system on a Vertisol in Central India. Soil \& Tillage Research, 76, 167-174. http://dx.doi.org/10.1016/j.still.2003.10.001

Raja Babu, C., Vijayalakshmi, C., \& Mohandass, S. (2005). Evaluation of rice (Oryza sative L.) genotypes for salt tolerance. Journal of Food, Agriculture and Environment, 3(1), 190-194.

Rathi, S., Bahruah, A. R., Chowdhury, R. K., \& Sarma, R. N. (2011). QTL analysis of seed dormancy in Indigenous rice of Assam, India. Cereal Research Communication, 39(1), 137-146. http://dx.doi.org/10.1556/CRC.39.2011.1.14

Saeidi, M. R., Abdolghaium, A., Hassanzadeh, M., Rouhi, A., \& Nikzad, P. (2008). Investigation of seed priming on some germination aspects of different canola cultivars. Journal of Food, Agriculture and Environment, 6(2), 188-191.

Singh, M., Bhattacharya, A. K., Nair, T. V. R., \& Singh, A. K. (2002). Nitrogen loss through subsurface drainage effluent in coastal rice field from India. Agricultural Water Management, 52, 249-260. http://dx.doi.org/10.1016/S0378-3774(01)00133-0

Vivek, K. T., Michael Gomez, S., Suresh, R., Satheesh Kumar, S., Yogameenakshi, P., Chezhian, P., \& Chandra Babu, R. (2004). Genetic diversity analysis among rice accessions differing in drought tolerance using molecular markers. Journal of Food, Agriculture and Environment, 2(3\&4), 217-222.

Yoon, C. G., Ham, J. H., \& Jeon, J. H. (2004). Mass balance analysis in Korean paddy rice culture. Paddy and Water Environment, 1, 99-106. http://dx.doi.org/10.1007/s10333-003-0018-z 